\title{
A Simple Proof of the Chuang's Inequality
}

\author{
Bikash Chakraborty
}

\begin{abstract}
The purpose of this paper is to present a short proof of the Chuang's inequality.
\end{abstract}

AMS Subject Classification (2010). 30D35

Keywords. Meromorphic function, small function, differential polynomial

\section{Introduction Definitions and Result}

Originally, the Chuang's inequality is a standard estimate in Nevanlinna theory but later on this inequality is used as a valuable tool in the study of value distribution of differential polynomials ([5]).

Recently, using this inequality, some sufficient conditions are obtained for which two differential polynomials sharing a small function satisfies the conclusions of Brück conjecture ([1], [2], [3], [4]).

At this point, we recall some notations and definitions to proceed further.

It will be convenient to let $E$ denote any set of positive real numbers of finite linear measure, not necessarily the same at each occurrence. Also we use $I$ to denote any set of infinite linear measure of $0<r<\infty$.

Let $f$ be a non-constant meromorphic function in the open complex plane $\mathbb{C}$. For any non-constant meromorphic function $f$, we denote by $S(r, f)$ any quantity satisfying

$$
S(r, f)=o(T(r, f)) \quad(r \longrightarrow \infty, r \notin E) .
$$

Now we recall the following definitions. 
Definition 1.1. A meromorphic function $a=a(z)(\not \equiv \infty)$ is called a small function with respect to $f$ provided that $T(r, a)=S(r, f)$ as $r \longrightarrow \infty, r \notin E$.

Definition 1.2. ([8])Let $n_{0 j}, n_{1 j}, \ldots, n_{k j}$ be non negative integers.

The expression $M_{j}[f]=(f)^{n_{0 j}}\left(f^{(1)}\right)^{n_{1 j}} \ldots\left(f^{(k)}\right)^{n_{k j}}$ is called a differential monomial generated by $f$ of degree $d\left(M_{j}\right)=\sum_{i=0}^{k} n_{i j}$ and weight $\Gamma_{M_{j}}=\sum_{i=0}^{k}(i+$ 1) $n_{i j}$.

The sum $P[f]=\sum_{j=1}^{t} b_{j} M_{j}[f]$ is called a differential polynomial generated by $f$ of degree $\bar{d}(P)=\max \left\{d\left(M_{j}\right): 1 \leq j \leq t\right\}$ and weight $\Gamma_{P}=\max \left\{\Gamma_{M_{j}}\right.$ : $1 \leq j \leq t\}$, where $T\left(r, b_{j}\right)=S(r, f)$ for $j=1,2, \ldots, t$.

The numbers $\underline{d}(P)=\min \left\{d\left(M_{j}\right): 1 \leq j \leq t\right\}$ and $\mathrm{k}$ (the highest order of the derivative of $f$ in $P[f]$ ) are called respectively the lower degree and order of $P[f]$.

$P[f]$ is said to be homogeneous if $\bar{d}(P)=\underline{d}(P)$.

$P[f]$ is called a Linear Differential Polynomial generated by $f$ if $\bar{d}(P)=1$. Otherwise, $P[f]$ is called Non-linear Differential Polynomial.

We also denote by $\mu=\max \left\{\Gamma_{M_{j}}-d\left(M_{j}\right): 1 \leq j \leq t\right\}=\max \left\{n_{1 j}+\right.$ $\left.2 n_{2 j}+\ldots+k n_{k j}: 1 \leq j \leq t\right\}$.

Now we are position to state the Chuang's inequality.

Theorem A. ([7]) Let $f$ be a non-constant meromorphic function and $P[f]$ be a differential polynomial. Then

$$
m\left(r, \frac{P[f]}{f^{\bar{d}(P)}}\right) \leq(\bar{d}(P)-\underline{d}(P)) m\left(r, \frac{1}{f}\right)+S(r, f) .
$$

In this paper, we give a short proof of the above inequality with some restrictions.

Theorem 1.1. Let $f$ be a non-constant meromorphic function and $P[f]$ be a differential polynomial. Then

$$
m\left(r, \frac{P[f]}{f^{\bar{d}(P)}}\right) \leq(\bar{d}(P)-\underline{d}(P)) m\left(r, \frac{1}{f}\right)+S(r, f),
$$

as $r \rightarrow \infty$ and $r \notin E_{0}$ where $E_{0}$ is a set whose linear measure is not greater than 2 . 


\section{Lemmas}

We prove the result, using the lemma of logarithmic derivative.

Lemma 2.1 (Lemma of Logarithmic Derivative). ([9]) Suppose that $f(z)$ is a non-constant meromorphic function in whole complex plane. Then

$$
m\left(r, \frac{f^{\prime}}{f}\right)=S(r, f) \text { as } r \rightarrow \infty \text { and } r \notin E_{0},
$$

where $E_{0}$ is a set whose linear measure is not greater than 2 .

Lemma 2.2. ([9]) Suppose that $f(z)$ is a non-constant meromorphic function in whole complex plane and $l$ is natural number. Then

$$
m\left(r, \frac{f^{(l)}}{f}\right)=S(r, f) \text { as } r \rightarrow \infty \text { and } r \notin E_{0},
$$

where $E_{0}$ is a set whose linear measure is not greater than 2 .

\section{Proof of Chuang's inequality}

Proof of theorem 1.1. Suppose that $P[f]=\sum_{j=1}^{t} b_{j} M_{j}[f]$ be a differential polynomial generated by a non-constant meromorphic function $f$. Further suppose that $m_{j}=d\left(M_{j}\right)$ for $j=1,2, \ldots, t$.

Without loss of any generality, we can assume that $m_{1} \leq m_{2} \leq \ldots \leq m_{t}$.

We have to prove the inequality (1.1) by induction on $t$.

If $t=1$, then in view of Lemma 2.2, the inequality (1.1) follows. Next we assume that the inequality holds for $t=l(\geq 2)$. Now we have to show that the inequality (1.1) holds for $t=l+1$.

For this, assume

$$
P[f]=\sum_{j=1}^{l+1} b_{j} M_{j}[f]=Q[f]+b M[f]
$$

where $Q[f]=\sum_{j=1}^{l} b_{j} M_{j}[f], M[f]=M_{l+1}[f]$ and $b=b_{l+1}$.

Then $m_{1} \leq m_{2} \leq \ldots \leq m_{l} \leq m_{l+1}$ and by hypothesis

$$
m\left(r, \frac{Q[f]}{f^{\bar{d}(Q)}}\right) \leq(\bar{d}(Q)-\underline{d}(Q)) m\left(r, \frac{1}{f}\right)+S(r, f) \text { as } r \rightarrow \infty \text { and } r \notin E_{0} \text {, }
$$


where $E_{0}$ is a set whose linear measure is not greater than 2 .

Thus

$$
\begin{aligned}
m\left(r, \frac{P[f]}{f^{\bar{d}(P)}}\right) & =m\left(r, \frac{Q[f]+b M[f]}{f \bar{d}(P)}\right) \\
& \leq m\left(r, \frac{Q[f]}{f^{\bar{d}(P)}}\right)+m\left(r, \frac{M[f]}{f \bar{d}(P)}\right)+S(r, f) \\
& \leq(\bar{d}(Q)-\underline{d}(Q)) m\left(r, \frac{1}{f}\right)+(\bar{d}(P)-\bar{d}(Q)) m\left(r, \frac{1}{f}\right) \\
& +(\bar{d}(P)-d(M)) m\left(r, \frac{1}{f}\right)+S(r, f) \\
& \leq(\bar{d}(P)-\underline{d}(P)) m\left(r, \frac{1}{f}\right) \\
& +(\bar{d}(P)+\underline{d}(P)-\bar{d}(Q)-d(M)) m\left(r, \frac{1}{f}\right)+S(r, f) \\
& \leq(\bar{d}(P)-\underline{d}(P)) m\left(r, \frac{1}{f}\right)+S(r, f)
\end{aligned}
$$

as $r \rightarrow \infty$ and $r \notin E_{0}$, where $E_{0}$ is a set whose linear measure is not greater than 2 , and $(\bar{d}(P)+\underline{d}(P)-\bar{d}(Q)-d(M))=m_{l+1}+m_{1}-m_{l}-m_{l+1} \leq 0$.

Thus by the principle of Mathematical Induction, the inequality follows.

\section{References}

[1] A. Banerjee and M. B. Ahamed, Meromorphic function sharing a small function with its differential polynomial, Acta Univ. Palack. Olomuc. Fac. Rerum Natur. Math., 54(1), (2015), 33-45.

[2] A. Banerjee and B. Chakraborty, On the generalizations of Brück conjecture, Commun. Korean Math. Soc., 31(2), (2016), 311-327.

[3] A. Banerjee and B. Chakraborty, Some further study on Brück conjecture, $A n$. Sţiinţ. Univ. Al. I. Cuza Iaşi Mat. (N.S.), 62(2)(f2), (2016), 501-511.

[4] A. Banerjee and S. Mallick, Brück conjecture - a different perspective, Commun. Fac. Sci. Univ. Ank. Sér. A1 Math. Stat., 65(1), (2016), 71-86.

[5] S. S. Bhoosnurmath, M. N. Kulkarni, and K. W. Yu, On the value distribution of differential polynomials, Bull. Korean Math. Soc., 45(3), (2008), 427-435.

[6] R. Brück, On entire functions which share one value CM with their first derivative, Results Math., 30(1-2), (1996), 21-24. 
[7] C. T. Chuang, On differential polynomials, in: Analysis of one complex variable, World Sci. Publishing, Singapore, 1987

[8] W. K. Hayman, Meromorphic Functions, Clarendon Press, Oxford, 1964.

[9] C. C. Yang and H. X. Yi, Uniqueness theory of meromorphic functions, Kluwer Academic Publishers, 2003.

Bikash Chakraborty

Department of Mathematics

Ramakrishna Mission Vivekananda Centenary College

Rahara

India-700 118

And

Department of Mathematics

University of Kalyani

Kalyani

India-741 235

E-mail: bikashchakraborty.math@yahoo.com, bikashchakrabortyy@gmail.com

Received: 22.02.2017

Accepted: 2.04 .2017 